\title{
A METHOD TO FORECAST SOLAR FLARES USING THE KISLOVODSK'S SOLAR PATROL OPTICAL TELESCOPE (SPOT)
}

\author{
Naga Varun Y. ${ }^{1}$, Paschenko M.P. ${ }^{2}$, Tlatov A.G. ${ }^{2,1}$, \\ Ageev S.S. ${ }^{1}$, Pakhalenko D.A. ${ }^{1}$ \\ ${ }^{1}$ Kalmyk State University, Elista, Russia \\ ${ }^{2}$ Kislovodsk Mountain Astronomical Station, RAS, Kislovodsk, Russia
}

\begin{abstract}
We demonstrate a method using solar patrol optical telescope's observations that help to forecast solar flares. The telescope continuously observes the Sun in various important wavelengths which are relevant for studying the solar chromosphere. However the most important amongst these spectral lines remains the ionized calcium resonant line CaII $K$ which gives valuable information about solar filaments, plages and other active chromospheric structures. By studying the actual varying spectral profile of plages we show that there exists a clear transformation of the profile into a particular specific type prior to the advent of solar flares. Such an analysis of spectroheliograms allows us to forecast flares in advance.
\end{abstract}

\section{DOI: 10.31725/0552-5829-2018-303-306}

The scientific task of the SPOT (Solar patrol optical telescope) is to observe on the full disk of the Sun various manifestations of solar activity, such as, plages, filaments, prominences, flares, nucleation of coronal mass ejections, and also magnetic fields of active and quiet regions of the solar atmosphere. SPOT continuously registers images of the full solar disc in CaII K $393 \mathrm{~nm}$, H-alpha $6563 \mathrm{~nm}$ spectral lines. In near future SPOT is going to be modernized in such a way that facilitates observing the Sun in He $1083 \mathrm{~nm}$ and magnetically sensitive Fe-I $630.15 \mathrm{~nm}$ and $630.25 \mathrm{~nm}$ spectral lines. The optical resolution of the SPOT telescope is about 1 arcsec for CaII $\mathrm{K}$ line. Images in the lines CaII, $\mathrm{H}$-alpha can be recorded once every three minutes. The most important feature of this telescope is that it can scan the Sun completely in a given interval of the wavelength and therefore making it possible to extract Dopplergrams from the initial data. In this way one can estimate the kinematic parameters of the eruption events like coronal mass ejections (CMEs). The telescope is designed to work continuously throughout the day in a semi automatic mode with a lifetime of 25 years.

Amongst all the above mentioned spectral lines one can observe active chromospheric features such as plages, filaments, chromospheric network and etc very well in the CaII $\mathrm{K}$ line which is a resonant spectral line. The intensity profile of CaII $\mathrm{K}$ is typical to that of any other line that is formed in the inner chromosphere and is shown in the fig. 1.

The reason why the profile of chromospheric lines such as CaII $\mathrm{K}$ is complicated is as follows: At first the profile of the spectral line very well maps with the temperature profile of the chromosphere. As one moves from left to 


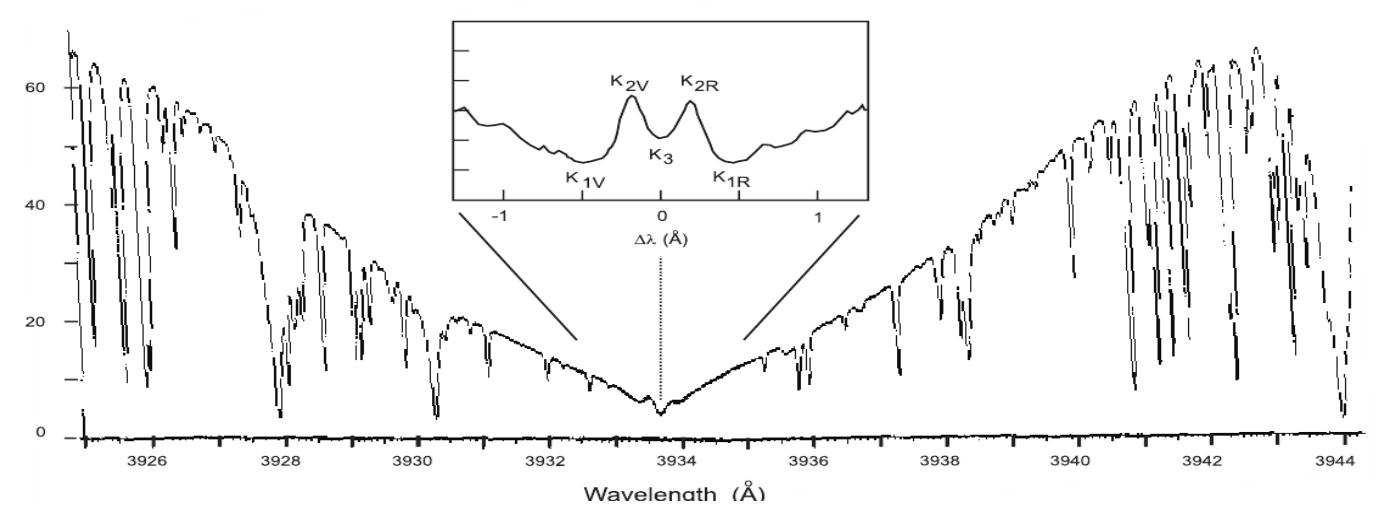

Fig. 1. Typical spectral profile of CaII K.

right in the fig. 3 one observes that at $\Delta \lambda<-0.4$ and to the left the opacity is considerably low and hence at these wavelengths we can observe intense photosphere. This is evedent from the fig. 1 where we observe that to the left of the Ca IIK core the intensity markedly increases. At $\Delta \lambda=-0.4$ the opacity allows

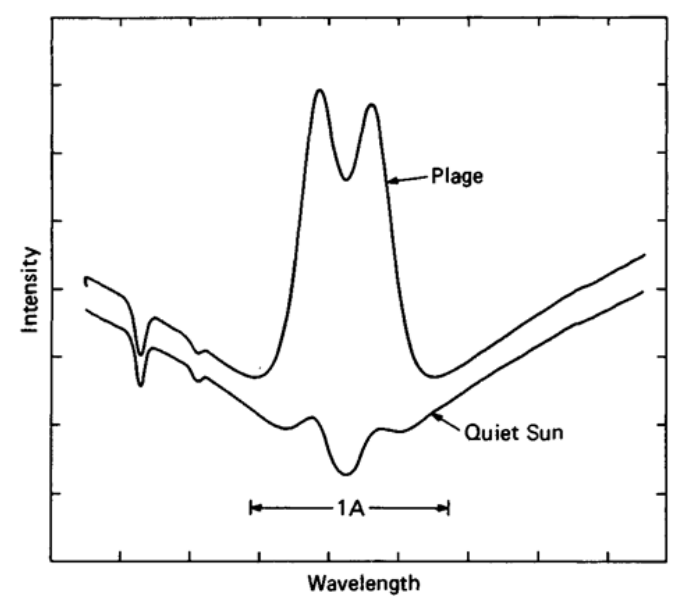

Fig. 2. CaII K profile at plages.

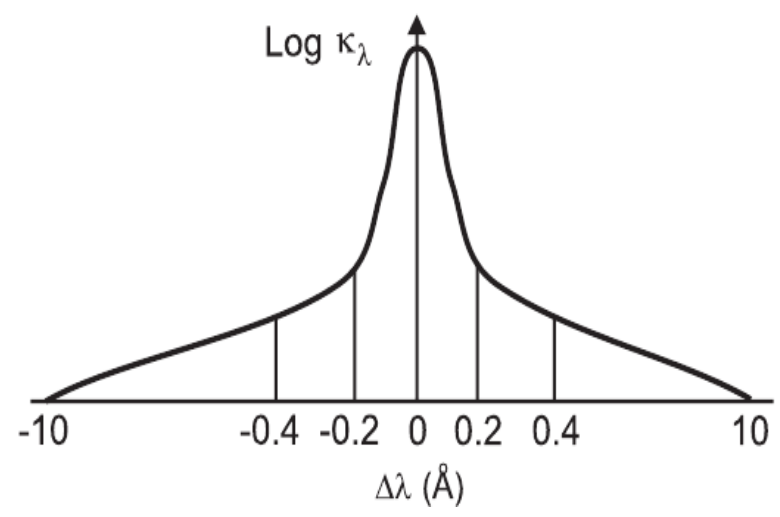

Fig. 3. Wavelength opacity function vs wavelength.

us to see only upto the temperature minimum region because of whch the intensity falls giving rise to the $\mathrm{K}_{1 \mathrm{~V}}$ minimum. This trend proceeds and at $\Delta \lambda=-0.2$ the opacity restricts our view only upto the middle chromosphere where temperature is considerably higher leading to a sharp rise in the intensity and giving rise to $\mathrm{K}_{2 \mathrm{~V}}$ local maximum. This one to one mapping between the spectral intensity profile and the temperature profile in the chromosphere breaks down at $\Delta \lambda=0$ or at the centre of the spectral line because the large opacity allows us to observe only the higher most layers of the chromosphere which are not in local thermal equilibrium (NLTE) [1]. The lack of local thermal equilibrium is mainly due to the lack of free electrons [3]. As a result the intensity abruptly falls and we observe the $\mathrm{K}_{3}$ dip. As we move further right in the fig. 3 we observe a similar qualitativey symmetric behaviour. However the $\mathrm{K}_{2 \mathrm{R}}$ intensity is a little less than the $\mathrm{K}_{2 \mathrm{~V}}$ because of the decreased Rayleigh 
scattering that allows us to go nearer to the temperature minimum region and hence the decreased intensity.

The spectral profile of CaII $\mathrm{K}$ at plages is quite similar but the only difference is that the $\mathrm{K}_{1 \mathrm{~V}}, \mathrm{~K}_{2 \mathrm{~V}}$ and $\mathrm{K}_{3}$ are quite pronounced. The enhanced intensity at plages is mainly due to its magnetic activity. As we see even at plages the breakup of local thermal equilibrium leads to the $\mathrm{K}_{3}$ dip.

Having explained the spectral profile of CaII $\mathrm{K}$ in the quite region and at plages we now explain how this profile changes prior to the advent of a solar flare. By considering fig. 4 , fig. 5 and fig. 6 we see that the $\mathrm{K}_{3}$ dip which is typical to the CaII $\mathrm{K}$ profile monotonically vanishes with time. These figures show the variations in the CaII $\mathrm{K}$ spectral profile at a point in the plage marked in the fig. 7. The X-axis in figs 4, 5, and 6 represent wavelength in pixel units and the $\mathrm{Y}$-axis represents intensity and the time is indicated in the upper left corner in each figure. We see that at $\mathrm{t}=7 \mathrm{~h}: 07 \mathrm{~m}: 46 \mathrm{~s}$ the spectral profile transforms into a Gaussian form. Quite interestingly after approximately 8 minutes we observe a flare which is clearly shown in the fig. 7.

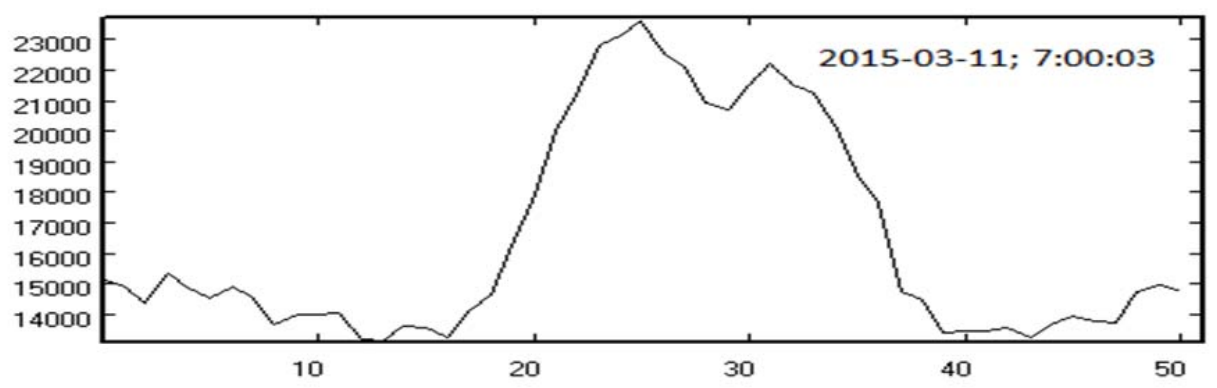

Fig. 4. Spectral profile at $\mathrm{t}=7 \mathrm{~h}: 00 \mathrm{~m}: 03 \mathrm{~s}$.

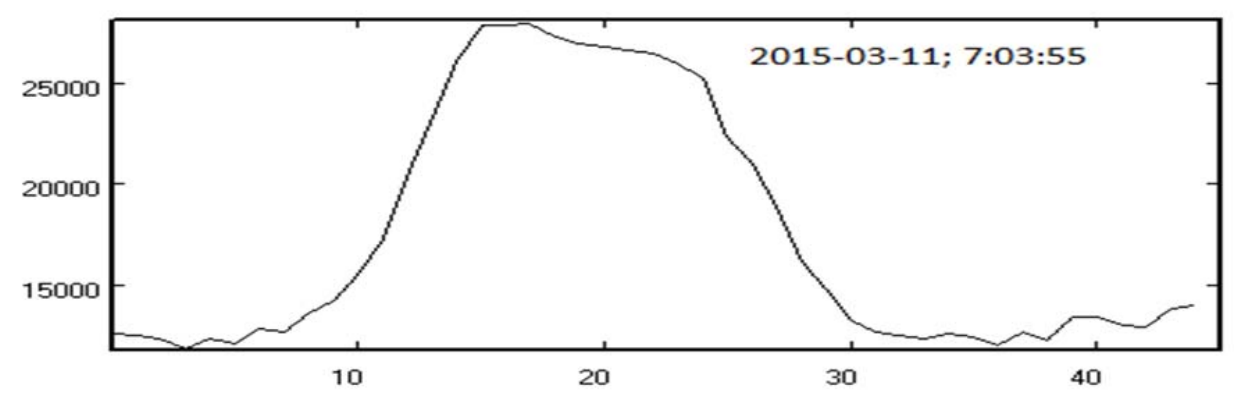

Fig. 5. Spectral profile at $\mathrm{t}=7 \mathrm{~h}: 03 \mathrm{~m}: 55 \mathrm{~s}$.

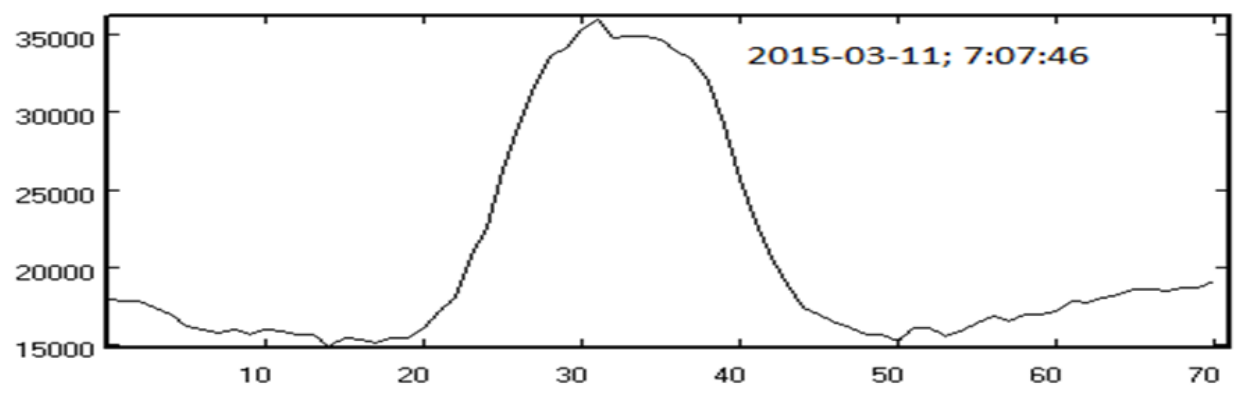

Fig. 6. Spectral profile at $\mathrm{t}=7 \mathrm{~h}: 07 \mathrm{~m}: 46 \mathrm{~s}$. 
The reason why the spectral profile is transformed into a Gaussian form is as follows: Prior to the solar flare there was an injection of electrons into the layers above the photosphere. The excessive injected electrons which happen to reach the higher layers of the chromosphere temporarily restore the local thermal equilibrium and therefore the mapping with the temperature profile is restored leading to the intense core centre of Ca IIK instead of a $\mathrm{K}_{3}$ dip.

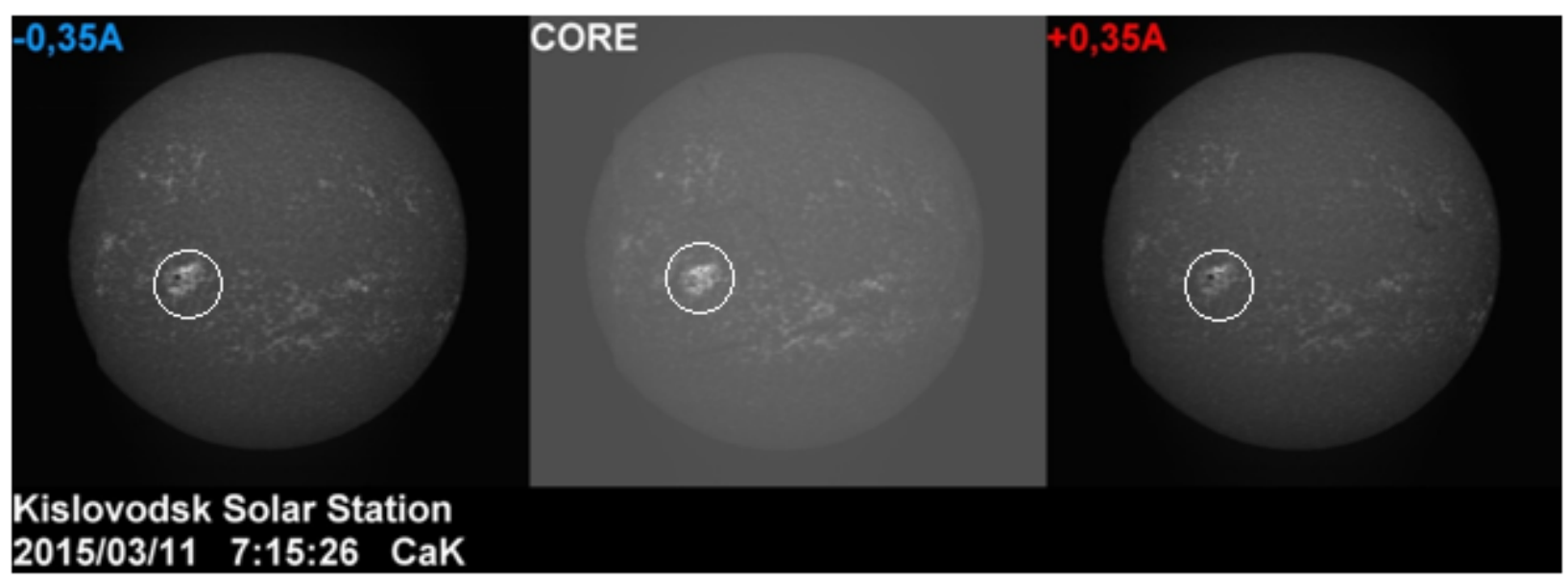

Fig. 7. Solar flare that occurred in the plage marked in a white circle.

We have analyzed many such events from the catalogue of Kislovodsk mountain station which dates from the year 2012 and found a similar behavior. Therefore this phenomenon of spectral profile's transition into a Gaussian form can be used as a diagnostic tool to forecast solar flares.

\section{Acknowledgments:}

1. We have taken the figures 1, 2 and 3 from the book [1] and highly recommend it for an in-depth understanding of the formation of spectral lines in the solar chromosphere.

2. This work is supported by the Russian science foundation grant (project no. 18-12-18000 and project no. 15-12-20001).

\section{References}

1. Antia H.M., Bhatnagar A., Ulmschneider P.: Lectures on Solar Physics. ISBN 3-54001528-0, 2003.

2. Aschwanden M. Physics of the Solar Corona: An Introduction with Problems and Solutions - Germany, Springer and Praxis publishing Ltd, P. 923, 2006.

3. Dolgarno A., Layzer D.: Spectroscopy of astrophysical plasmas. ISBN 0521263158 , 2004. 